\title{
Lateral Entry Guidance for Lunar Return Vehicles
}

\author{
Zunshi Shui (Corresponding author) \\ Institute of Precision Guidance and Control, Northwestern Polytechnical University \\ PO box 250, Xi' an 710072, China \\ E-mail: shuizunshi@yahoo.com.cn
}

Jun Zhou

Institute of Precision Guidance and Control, Northwestern Polytechnical University

Xi'an 710072, China

Zhilei Ge

Institute of Precision Guidance and Control, Northwestern Polytechnical University

Xi'an 710072, China

Received: January 9, 2012 Accepted: February 10, 2012 Published: March 1, 2012

doi:10.5539/mas.v6n3p100

URL: http://dx.doi.org/10.5539/mas.v6n3p100

\begin{abstract}
A lateral entry guidance is designed based on azimuth error and crossrange error for a low $\mathrm{L} / \mathrm{D}$ ratio lunar return vehicle. The conventional technique to determine the bank sign is according to the crossrange error, which might cause large crossrange deviation during Kepler phase if azimuth error at skip out point is large. This paper develops a combined lateral guidance logic to minish accumulate crossrange error caused by azimuth error during Kepler phase. The lateral logic decides the value of crossrange threshold by constantly predicting the crossrange at skip out point. The azimuth error at skip out point is regulated to a small value by only one bank reverse through online adjusting reversal threshold. The effect of earth rotation is compensated by moving the landing site to opposite direction. During the second entry, the lateral logical is designed based on the crossrange error to achieve precise lateral control. The lateral guidance logic is validated by numerical simulations. Monte Carlo simulations show that the proposed lateral guidance logic can deliver the vehicle to the desired landing site in the presence of large initial dispersions and disturbance.
\end{abstract}

Keywords: Entry guidance, Skip entry, Lateral guidance, Lunar return

\section{Introduction}

The atmosphere entry of lunar vehicle is very crucial for the hard environment. The vehicle approaches the atmosphere at $11 \mathrm{~km} / \mathrm{s}$ in velocity. The high speeds associated with reentry will create high g-loads and heat loads, meanwhile for a low lift-to-drag (L/D) vehicle, a skip entry trajectory is needed to achieve long downrange. The lunar return vehicle is bluntly and has a low L/D ratio which is usually less than 0.5 (e.g., the L/D of Apollo is about 0.3). If the downrange is long, the vehicle will skip out the atmosphere, where has a long ballistic flight with neglectable controllability. According to the former reason, a high performance of lateral guidance is demanded for successfully guiding the vehicle to desired landing site.

The entry guidance has been extensively studied and numerous methods have been proposed (Rea J. R. \& Putnam Z. R., 2007; Brunner C. W. \& Lu P., 2010). Most researches focus on the longitudinal guidance, such as shuttle entry guidance based on the drag-to-velocity profile (Harpold J. C. \& Graves C. A., 1979), the quasi-equilibrium Glide guidance (Shen Z. J. \& Lu P., 2003), etc. Little attention is paid to the lateral guidance. There are mainly three methods of lateral guidance as follows:

1) Bank reverse according to the crossrange error. (Lickly D. J. \& Morth H. R., 1963).

2) Bank reverse depending on the azimuth error, which is the error angle between heading angle and azimuth angle (Harpold J. C. \& Graves C. A., 1979). 
3) Determined by the tracking law when simultaneous tracking of longitudinal and lateral reference profiles ( $\mathrm{Lu}$, P., 1999).

Among these three methods, the last method is designed for medium or high lift-to-drag (L/D) ratios vehicles. The blunt vehicle studied in this paper has a low L/D ratio, which has limited maneuverability. It is difficult to track the longitudinal and lateral reference profiles simultaneity. Besides during entry the main critical constrains such as range, energy are only concerned with longitudinal motion. Hence the reference lateral profiles do not have to be tightly tracked, This paper will focus on only the method 1 and 2 .

The paper is organized as follow. Section 2 analyses the lateral entry guidance based on the crossrange error and azimuth error respectively. Section 3 designs lateral entry guidance, which is suitable for lunar return vehicles. Section 4 presents the longitudinal guidance in subsequent simulation. Section 5 gives simulation results and discussion. Concluding remarks are given in the final section.

\section{Bank Reversal Logic}

\subsection{Conventional Based on Azimuth Angle Error}

When the vehicle penetrates into atmosphere, the angle of attack is determined by trim ability of the vehicle. With the given mach number and altitude, the angle of attack can be calculated. The slide bank angle is zero theoretically. As a result, the mainly control variable is bank angle, which is defined as the amount of the vehicle rotation about its velocity vector.

The conventional bank reversal criterion is based on the pre-specify crossrange error threshold. The value of threshold shrinks with the decrease of velocity. A typical crossrange error threshold is shown in Figure 1. Whenever the crossrange along the trajectory exceeds the threshold, the sign of bank angle is reversed. This method directly controls crossrange error, and leads to high precise landing.

\subsection{Bank Reversal Logic Based on Azimuth Angle Error}

Supposing the coordinates of the vehicle's current location are $(\lambda, \phi)$, the azimuth angle $\sigma_{t}$ is defined as angle between north and the line-of-sight with respect to the landing site $\left(\lambda_{T}, \phi_{T}\right)$ along the great circle. The value of $\sigma_{t}$ is represented by

$$
\tan \sigma_{t}=\frac{\sin \left(\lambda_{T}-\lambda\right)}{\cos \phi \tan \phi_{T}-\sin \phi \cos \left(\lambda_{T}-\lambda\right)}
$$

Let azimuth error be $\Delta \sigma=\sigma_{T}-\sigma_{t}$, where $\sigma_{T}$ is velocity azimuth angle.

If azimuth error excesses the pre-specify boundary, the lateral guidance reverse the direction of bank angle, otherwise, the sign of bank angle keep unchanged. The underlying concept of this method is making the vehicle fly directly to the landing site. But the heading error always exhibits large and fast variation as the trajectory approaches the landing site (Shen Z. J. \& Lu P., 2004), which makes it undesirable for choosing lateral guidance logic parameters.

The criteria of choosing threshold parameters for both lateral logics mentioned above is that the crossrange satisfy final position demand without excessive reversal.

\section{Improved Lateral Guidance Logic}

The lunar return vehicle may require flying a downrange as long as $10000 \mathrm{~km}$, so the effect of earth rotation and crossrange error accumulation in Kepler phase must be taken into consideration. In order to handle these problems, this paper takes some special measures which will be discussed in detail as follow.

\subsection{The Effect of Earth Rotation}

During a long time flight, the effects of earth rotation may cause the vehicle deviate too large a crossrange error at the start of the final phase, which exceeds the capability of the final phase guidance can compensate for and causes an unaccepted lateral error.

For a fixed reentry mission scenario, the crossrange caused by earth rotation changes slightly. The lateral logic can use bias landing site technique to account for the effect of earth rotation, which moves the landing site from the true landing site to a specified bias along the direction of crossrange. The changed landing site will influence the crossrange error, thus force the vehicle flying to the desired direction during Kepler phase. The biased landing site is only used for lateral guidance logic, while the longitudinal logic still use true landing site to generate bank command. A schematic biasing is shown in Figure 2.

The amount biased the true landing site can be obtained as follow (Brunner C. W., 2008) 


$$
\left[\begin{array}{cc}
\frac{\cos \left(\lambda_{T}-\lambda\right) \cos \phi_{T}}{\sin \left(s_{\text {togo }}\right)} & -\frac{\sin \left(\lambda_{T}-\lambda\right) \sin \phi_{T}}{\sin \left(s_{\text {togo }}\right)} \\
-\cos \phi_{T} \cos \phi \sin \left(\lambda_{T}-\lambda\right) & \sin \phi \cos \phi_{T}-\sin \phi_{T} \cos \phi \cos \left(\lambda_{T}-\lambda\right)
\end{array}\right]\left[\begin{array}{c}
\delta \lambda \\
\delta \phi
\end{array}\right]=\left[\begin{array}{c}
\delta \Psi \cos \Psi \\
0
\end{array}\right]
$$

where $\delta \lambda$ and $\delta \phi$ are the change of longitude and latitude respectively. $\delta \Psi$ can be determined through simulation results.

\subsection{The Crossrange Caused by Azimuth Error}

As mentioned previously, the vehicle will skip out of the atmosphere and maintain a long ballistic flight. If the angle between velocity and line-of-sight is large, the crossrange will increase with time elapse. At the same time, it may cause a large crossrange deviation at the start of the final phase. However, this large deviation may also excess the vehicle's compensate ability. Thus, the lateral guidance logic must consider azimuth error at the beginning of Kepler phase simultaneity.

Defined the crossrange variable $x$ as

$$
x=\sin ^{-1}\left[\sin \left(s_{\text {togo }}\right) \sin (\sigma-\Psi)\right]
$$

All variables have units of radians. Obviously small heading error means small crossrange error. In order to confine the crossrange deviation to an acceptable bound, we can restrict heading error to a small amount. The procedure will be given subsequently.

In the actual flight, instantaneous bank angle reversals are impossible. If a bank reverses from above, it will slow down the energy depletion; on the contrary, if a bank reverses from below, it will speed up the energy depletion. An increase in the number of roll reversal is undesirable because each reversal causes a deviation from the reference trajectory profile as well as costs additional fuel. In addition, the downrange is sensitive to the velocity and exit angle at skip out point. Therefore the effects of bank reversals on the trajectory should be minimized.

Theoretically, one bank reversal is enough to control the lateral error. In order to control the azimuth angle error at skip out point by only one bank reversal, a dynamic lateral threshold has to be designed.

The lateral threshold is assumed as a constant in a guidance cycle. In the next guidance cycle, the lateral guidance will constantly predict the azimuth angle error at skip out point. Based on the difference between predicted and desired value, the value of the lateral threshold is adjusted. Eventually meet the requirement of azimuth angle error.

Supposed in one scenario, the crossrange is shown in dash line in Figure 3. We can see the value of threshold is too small, the moment of bank reversal is too early thus a positive crossrange is caused. According to equation (3), the azimuth angle is undesirable too, and then the threshold is increased in next guidance cycle, which makes the vehicle aligning the landing site at skip out point. Through online adjusting reversal threshold, the azimuth error is maintained small.

\section{Longitudinal Guidance}

The longitudinal guidance employs numerical predictor-corrector guidance. The guidance assumes a constant bank angle trajectory. Firstly, downrange is predicted using current bank angle; Secondly, comparing the predicted downrange with desired downrange, a new bank angle could be obtained. The corrector based on the following concept, if the vehicle undershoots the final position, the magnitude of bank angle should be decreased, which will result in more lift vector in the vertical plane making the vehicle stay in the air longer and increase its range, vice versa.

\section{Simulation}

The vehicle model used in this paper is the Apollo lunar vehicle. The vehicle has a capsule configuration with a $\max \mathrm{L} / \mathrm{D}=0.3$. The overload during entry is less than $8 \mathrm{~g}$ and the entry interface is $120 \mathrm{~km}$ in altitude and $11 \mathrm{~km} / \mathrm{s}$ in velocity with flight path angle -5.7 degree. The guidance cycle is 1 second. The desired downrange is 10000 $\mathrm{km}$.

In Figure 4, the bank angle is tracked to the command bank angle tightly. The plot clearly shows that there is only one bank reversal during first entry to skip out. In Figure 5 the crossrange relative to the true landing site is presented. From Figure 5, it can be seen that the crossrange meet the final crossrange error requirement.

To evaluate the robustness and performance of the proposed guidance logic, a series of simulations are performed. The dispersions in entry interface state and other parameters for Monte Carlo simulation are given in 
Table 1.

The simulation results of 500 dispersed cases are shown in Figures 6 8. In Figure 6 the landing error of 500 cases are shown with 497 cases landing within $7 \mathrm{~km}$ of landing site and with 3 cases failure. Figure 7 shows the azimuth error respect to the bias landing site before skip out the atmosphere. We can see the azimuth error at skip out point is confined to $0.015 \mathrm{rad}$. The corresponding crossrange respect to bias landing site is given in Figure 8 .

\section{Conclusion}

A new lateral guidance logic considering azimuth error at skip out is presented for long lunar return. The accumulated crossrange error during Kepler phase is minished by controlling the azimuth error. The lateral guidance logic employs only one bank reversal, which will help alleviate the disturbance of bank reversal dynamic, as well as save fuel. The effect of earth rotation is compensated by moving the landing site to a biased site. Precise control of crossrange error provides a good entry condition for the second entry, which will increase the probability of success entry. The validation of proposed logic is done through extensive testing. Simulation results show that the proposed lateral guidance is robust to dispersion.

\section{References}

Bairstow, S. H., \& Barton, G. H. (2007). Orion reentry guidance with extended range capability using PredGuid. AIAA Guidance, Navigation and Control Conference and Exhibit, Hilton Head, South Carolina, 20-23.

Brunner, C. W. (2008). Skip entry trajectory planning and guidance. Iowa: Iowa State University.

Brunner, C. W., \& Lu, P. (2010). Comparison of numerical predictor-corrector and apollo skip entry guidance algorithms. AIAA Guidance, Navigation, and Control Conference, Toronto, Ontario Canada, 1-20.

Harpold, J. C., \& Graves, C. A. (1979). Shuttle entry guidance. The Journal of the Astronautical Sciences, 37(3), 239-268.

Lickly, D. J., \& Morth, H. R. (1963). Crawford B S. Apollo entry guidance. Massachusetts Inst. of Tech. NASA-CR-52776.

Lu, P. (1999). Regulation about time-varying trajectories: precision entry guidance illustrated. Journal of Guidance, Control, and Dynamics, 22(6), 784-790. http://dx.doi.org/ 10.2514/2.4479

Rea, J. R., \& Putnam, Z. R. (2007). A comparison of two orion skip entry guidance algorithms. AIAA Guidance, Navigation and Control Conference and Exhibi, Hilton Head, South Carolina, 1-19.

Shen, Z. J., \& Lu, P. (2003). Onboard generation of three-dimensional constrained entry trajectories. Journal of Guidance, Control and Dynamics, 26(1), 110-121. http://dx.doi.org/ 10.2514/2.5021

Shen, Z. J., \& Lu, P. (2004). Dynamic lateral entry guidance logic. AIAA Guidance, Navigation, and Control Conference and Exhibit, Providence, Rhode Island, 1-18.

Table 1. The class and level of variation

\begin{tabular}{|c|c|}
\hline State/parameter & $3 \sigma$ value \\
\hline Longitude(deg) & $1.7967^{\circ}$ \\
\hline Latitude(deg) & $0.8983^{\circ}$ \\
\hline Velocity $(\mathrm{m} / \mathrm{s})$ & $100 \mathrm{~m} / \mathrm{s}$ \\
\hline Flight path angle(deg) & $0.1^{\circ}$ \\
\hline Heading angle $($ deg $)$ & $0.1^{\circ}$ \\
\hline CL and CD & $15 \%$ \\
\hline Mass & $5 \%$ \\
\hline Atmospheric density & $30 \%$ \\
\hline
\end{tabular}




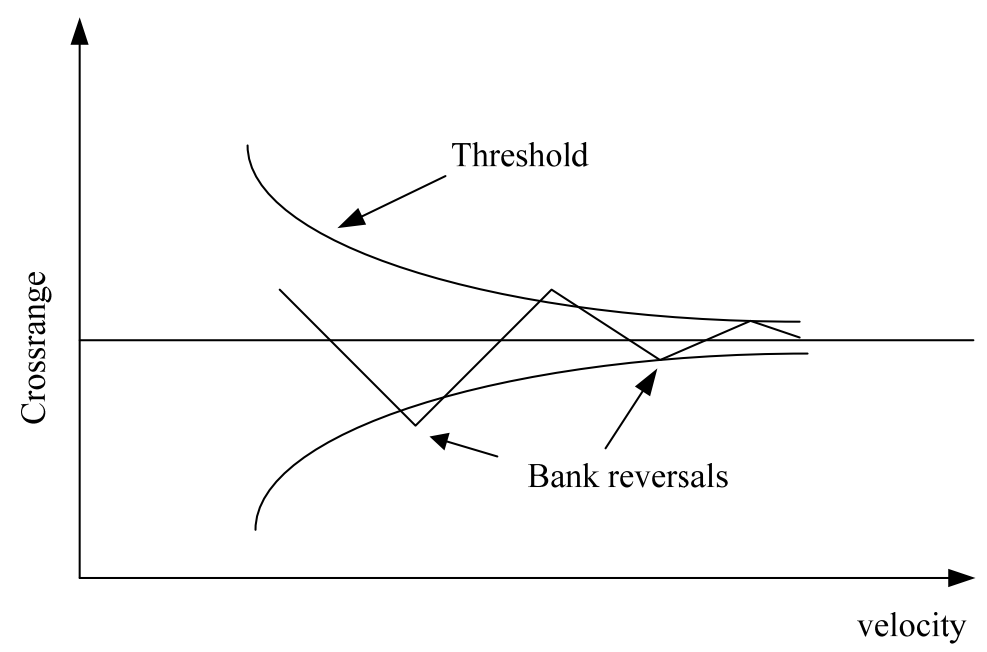

Figure 1. A typical crossrange error threshold

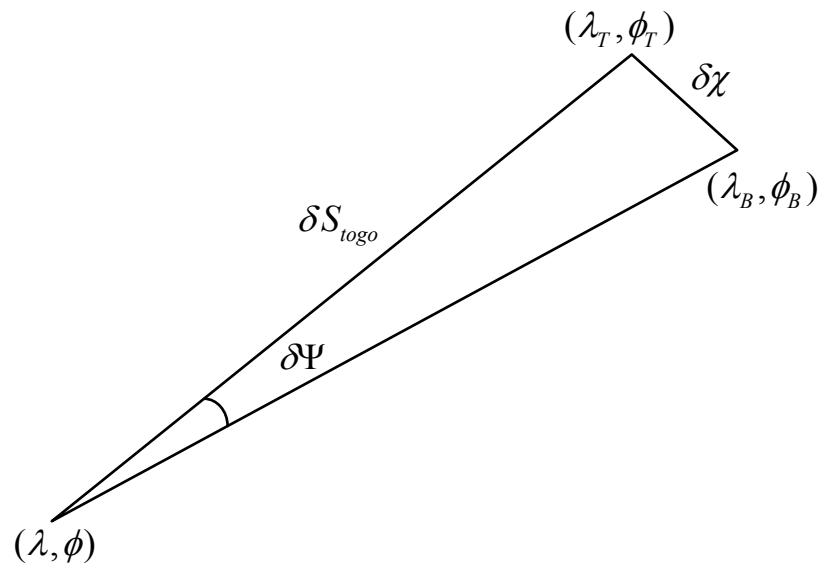

Figure 2. A schematic biasing

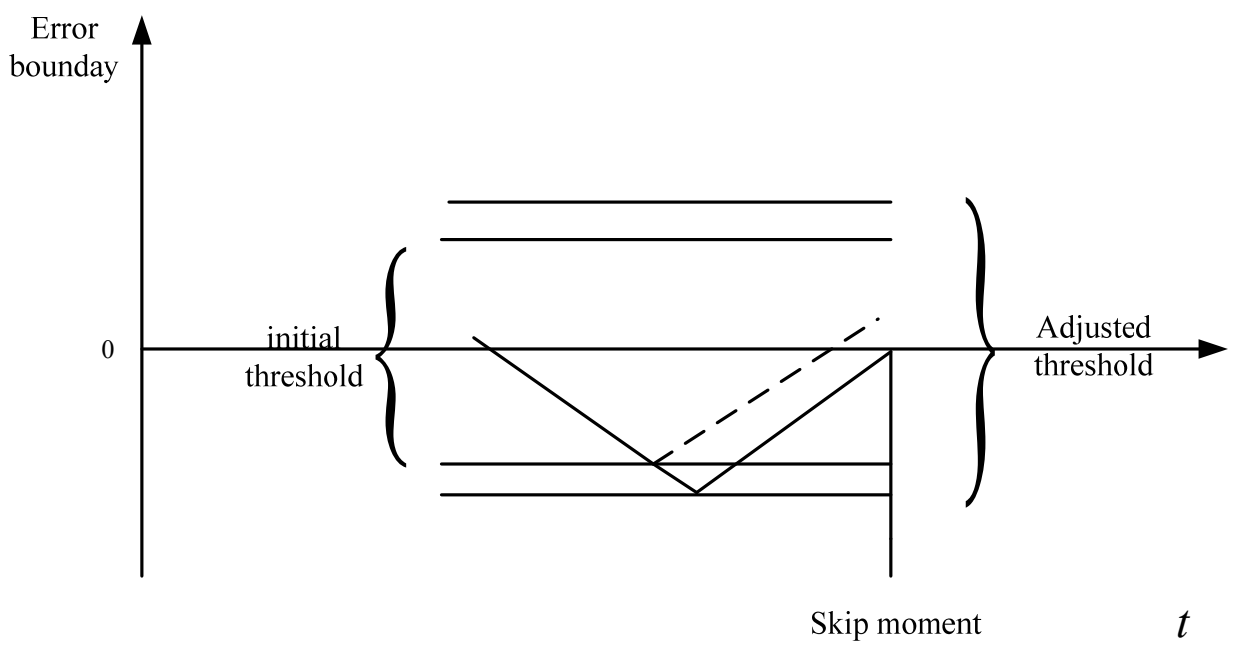

Figure 3. Geometric illustration of the threshold adjusted for the bank reversal 


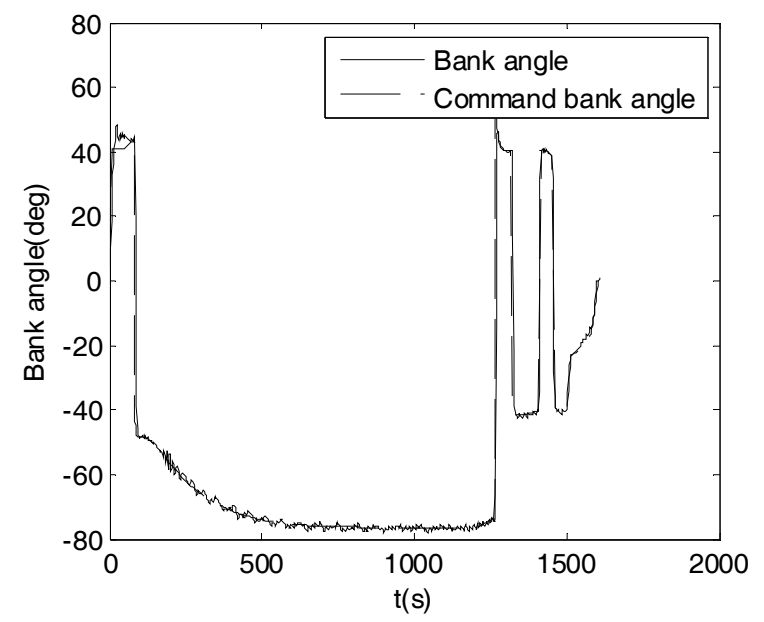

Figure 4. Command bank angle and actual bank angle profiles

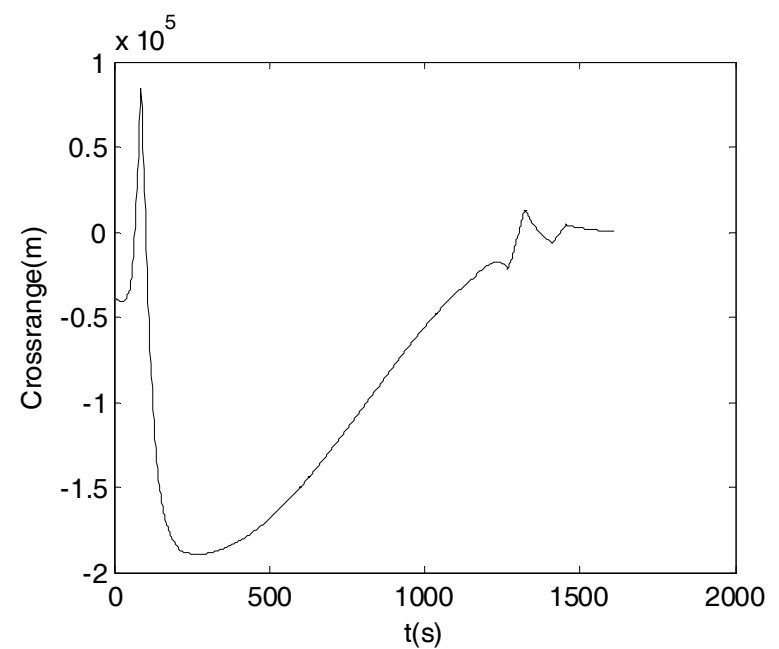

Figure 5. The crossrange relative to the true landing site

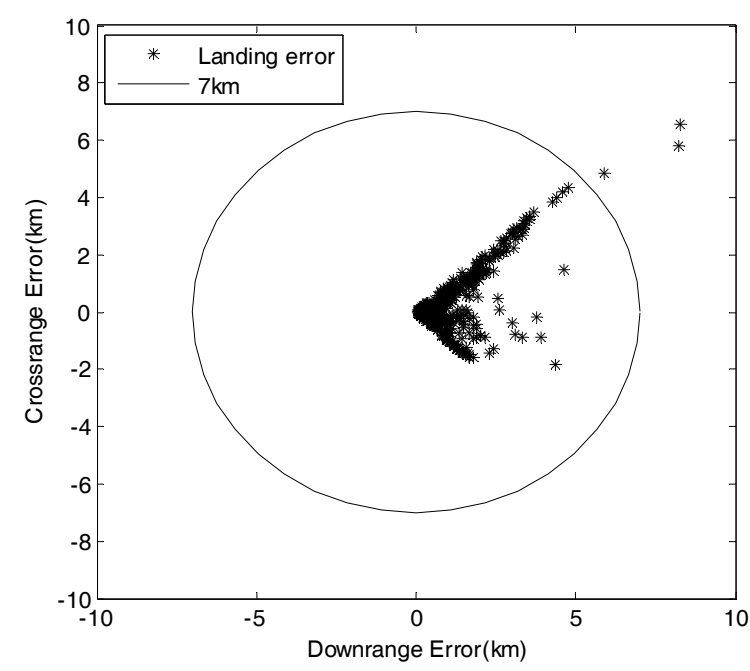

Figure 6. Landing errors for 500 dispersion cases 


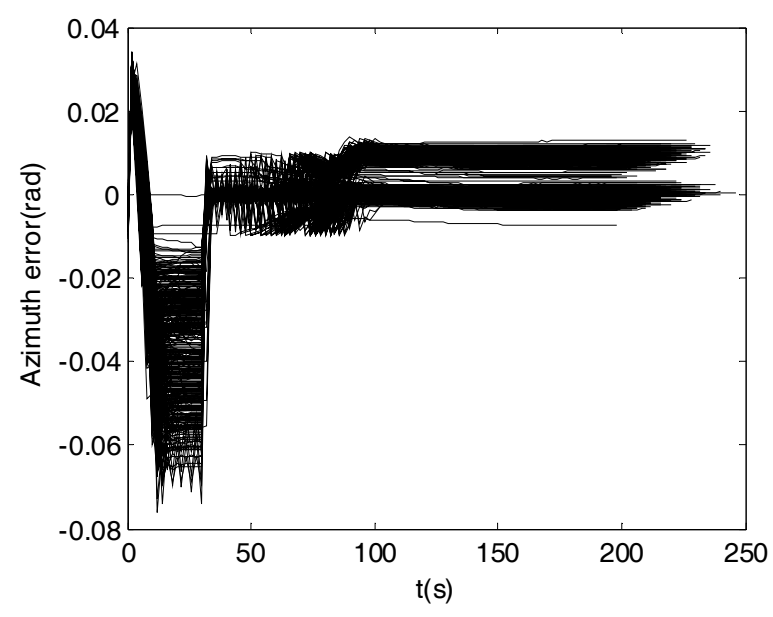

Figure 7. Azimuth errors for 500 dispersion cases

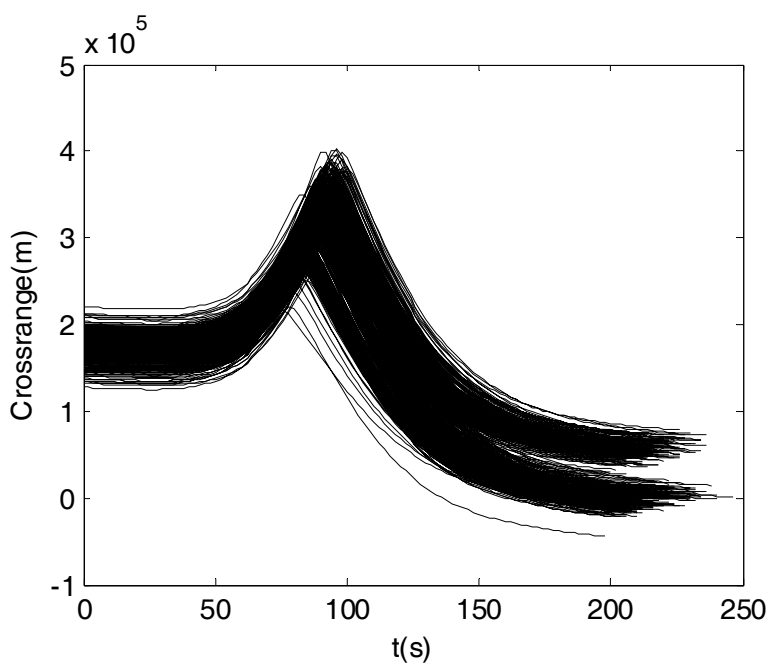

Figure 8 . The crossrange relative to the biased landing site 